Paediatrica Indonesiana 15 : 297 - 302. Nov. - Dec. 1975. 297

From the Department of Chest Diseases, Medical School, University of Indonesia/"Persahabatan" Hospital, Jakarta

\title{
Treatment of In-Patient Pulmonary Tuberculosis
}

$$
\text { by }
$$

RASMIN RASJID

\begin{abstract}
The progress of a tuberculous lesion was discussed. A treatment regimen of pulmonary tuberculosis in the warnds was layed out, especially for the "Persahabiatan" Hospital, Jakarta.

Rifiampicin seems to be a potent drug. However, for developing countries like Indonesia it is still very expensive. Possibly a combinations of $I N H+R M P+E B$ will be most effective at present.

Only a daily treatment is carried out in "Persahabatan" Hospital.

* Invited paper at the Third National Indonesian Paediatric Congress, Surabaya, July 1 - 6, 1974 .

Received 11 th. September 1974.
\end{abstract}




\section{Introduction}

Many anti-tuberculous drugs, antibiotics as well as synthetic drugs, have been discovered since Waksman (1942) introduced streptomycin. Since then chemotherapy becomes the basic treatment for pulmonary tuberculosis.

In the beginning bacteriostatics, but later on also bactericidal dirugs were introduced. It was hoped that these latter would be able to destroy the tuberculous bacilli in a relatively short time.

However, up to now treatment of pulmonary tuberculosis still lasts for a long time. The average duration of treatment will still be 2 to 3 years, with another 2 to 3 years of observation. This continuous management will frustrate the patient so that he usually will give up the treatment on account of his finances, or he will see another doctor, who is thought to be able to cure him within several weeks only. The treatment becomes then irregular and incomplete, worsening the condition which becomes more difficult to be properly treated.

Lack of knowledge in the management also results in an incomplete healing. In treating lung tuberculosis every doctor should know the following :

1. The progress of tuberculous lesion and the methods of its healing.
2. The scheme of treatment.

3. The right drugs for the treatment.

Chemotherapy is often given to cases, which in fact must be operated on or is contimued in already healed cases.

We seldom get early cases in our Department of Chest Diseases ; the majority are retreatment cases.

The lung tuberculosis begins with an exudative lesion, a small pneumonic infiltrate. After a certain period, the infiltrate will follow one of the 3 following ways :

1. Re-absorption, resulting in complete disappearance.

2. Formation of fibrosis resulting in improvement leaving cicatrices or calcified foci.

3. The infilitrate will expand intensively, followed by formation of necrotic and caseous mass, which later on liquifies, leaving ultimately a cavity behind.

Gradually, a thick wall will be formed around the cavity.

From the early formed cavity as well as from a thick wall one new exudative lesion may exist, which will develop again as mentioned above. Currently, a new exudativo lesion can be formed from the cavity as well as from the wall progressing in the similar pattern as the primary cavity.

Non-sclerotic cavity can also exist being clean outside as well as 
inside and is called a clean-up cavity. It can remain as clean - up cavity (open healling) or can also become a cicatrix (scar healing).

Not all exudative lesions becoming necrotic will form a cavity. Some encapsulate themselves into the so called nodular lesion or tuberculoma. This can reactivate and form also a cavity inside and gradually getiting a thick wall as well. The nodular lesion may become inactive and form ultimately an encapsulated healed rest.

From the above we can distinguilsh three kinds of lesion, i.e. :

1. Exudative, which is still active requiring an active treatment.

2. Lession that tends to heal still needing supportive treatment, so that it realiy becomes inactive.

3. Healed lesion requiring no treatment at all.

Another feature is the sputum conversion ; if it occurs it gives good hope for a total recovery.

In respect wtith treatment, lung tuberculosis can be categorized as follows :

1. Lung tuberculosis, which can be cured by chemotherapy only. First line drugs only as well as second line drugs thereafter can give good results. The healing can be restitutio ad integrum, or in the form of leaving some fibrotic changes.

The most important things to remind are :
1.1. An intensive initial treatment ;

1.2. A regular and adequate chemotherapy ;

1.3. To prevent defaulters.

By implementing these precautions it is hoped that this category will not proceed to the following categories 2 or 3 .

2. Lung tuberculosis, which must be operated on after unsuccessful chemotherapy.

3. Other forms of lung tuberculosils. These are far advanced cases, which will not be cured completely by chemotherapy, and which are too late for surgical intervention. Usually the Iung function of these cases is already very poor.

The main efforts for these cases should be :

3.1. To keep the sputum negative.

3.2. To keep an optimal general condition.

3.3. To prevent drug intoxication. Sometimes an operation is performed in these cases, just to relieve existing sufferings.

What kind of drugs should be given? There is no definite regimen. Various combinations of drugs should be tried to these cases.

There is also no definite time schedule in administering the drugs. 
Usually it will last for a very long time.

In general, patients visiting the Department of Chest Diseases "Persahabatan" Hospital, Jakarta, are rotreatment cases. They have already been treated by several doctors, underwent irregular and incomplete treatment, so that exudative lesions combined with fibrotic or cirrhotic changes are observed, usually with a cavity; the sputum is frequently positive and the bacilli are usually allready resistant to many drugs.

Besides the general management (bedrest, adequate diet) the patient is treated with anti-tuberculous drugs as follows :

1. First we apply the first line drugs, i.e. INH, streptomycine and PAS. Reasons why to give first line drugs are :

1.1. inexpensive.

1.2. minimal side-effects.

1.3. on admission sensitivity tests are not yet obtainable.

1.4. lack of a complete previous medical record.

1.5. improvement (clinically as well as radiologically) is still] possible with these first line drugs based on our own experience.

1.6. these drugs are mainly available in government's hospuitails.
2. Since the very start we have to decide, whether therapy will consist of drugs only, or whether it will be followed by surgical intervention.

3. Kanamycin and ethambutol are the next drugs to be used, in the following combinations :

3.1. Kanamycin + INH + PAS. 3.2. Fithambutol + INH + PAS. 3.3. Kanamycin + $\mathrm{INH}+\mathrm{Et}$ hambutol.

We often use the last combination in case of a lung operation. Reasons to choose kanamycin and ethambutol right after the finst line drugs are :

- they give a reasonable good result

- toxicity is low

However, those drugs are rather expensive, and usually not available in government's hospitals.

4. The next drug to be used is rifampicion.

5. We seldom use other drugs like thiacetazone, cycloserine, ethionamide, pyrazinamide etc. However, on certain rare indications we also use these drugs.

Reasons, why we seidom use these drugs are :

5.1. normally, drugs mentioned earlier give good results.

5.2. the latter drugs can cause serious side effects. 
We still use PAS initially for patients who cannot afford to buy other expensive drugs. We understand that the better the condition of the partient becomes, the more the patient dislikes swallowing PAS tablets. We then soon replace PAS with ethambutol which gives a similar effect in the combination with INH and streptomycin.

Various combinations can be used. Many investigators have published their results with different combinatilons. Some investigators mention defined combinations for specific kindls of lung tuberculosis. In this context, Japanese scientilsts have published their results in "Controlled trials of chemotherapy of tuberculosils 1957-1966" issued by the Cooperaltive Study Unit on Chemotherapy of Tuberculosis of the National Sanatoria, Tokyo National Chest Hospital, Tokyo, Japan, 1970.

At our departmen't such trials have not been done yet. Therefore, regimens described above could not be mentioned as the best. For the time being those regimens are still being used because of :

1. Favourable results are observed up to now

2. Toxicities are very rare

3. The drugs are very easily to be obtained, so that continuity of treatment can be guaranteed.

In curing lung tuberculosis some principles must be taken into account :
1. Examine the patient thoroughly to get the secure diagnosis; $x$-ray findings, sputum examinations, etc. should be cautiously evaluated.

2 Prevent the patient to be a defaulter, explain to him the advantages of taking medicine for a long period.

3. The physician should carry out the treatment precisely accordling to the regimen.

4. He should carry on the intensive initial chemotherapy.

Other items to be considered in sellecting chemotherapy are :

1. The M.I.C. of various drugs.

The M.I.C. of $\mathrm{Tb}, 1$ is reasonable, i.e. $1.2 \mathrm{mcg} / \mathrm{ml}$; however, sideeffects if present, are very severe.

2. The possibililty of cross res:stance.

3. Drugis with synergic reaction will be advanitageous.

The synergic reaction between rifampicin and ethambuitol is well established.

It is suggested that in the future $\mathrm{INH}+\mathrm{RMP}+\mathrm{EB}$ will be the combination of choice.

4. Blood level of the drug is also important.

For instance, Streptomycin will reach a level of $30-40 \mathrm{mg} / \mathrm{ml}$ in 30 minutes, which will come down gradually until it disappears after 6 hours. 
This is also applicable to INH and other drugs. In administering INH 3 times a day, 3 tops will be obtained, while once a day will give only one top.

It has been stated that rifampiain is able to remain in the blood for 12 hours.

Other drugs disappear completely after 6 hours.

5. Atlention must also be paid to possibile side-effects.

Not a 11 patients will inform the doctor spontaneously, because of :

5.1. ignorance of side-effects

5.2. he regards his doctor knowi everything.

In general the dosage used is based on $\mathrm{mg} / \mathrm{kg}$ body-weight, e.g. INH 5-7 mg/kg body-weight (on the average $300-400 \mathrm{mg}$ a day), PAS $200 \mathrm{mg} / \mathrm{kg}$ body-weight, ethambutol 15-25 $\mathrm{mg} / \mathrm{kg}$ body-weight, etc. ; Streptomycin is given 1 gram a day maximally. The drugs are administered every day. In the wards intermittent therapy is abandoned.

The basic treatment of lung tuberculosis has not yet changed in the last 10 years. The period of sputum. convension is normally used to evaluate whether there will be a relapse or not. The shorter the period the less chance for a relapse.

It is known that INH + RMF produces the shortest conversion period. But it does not mean that the duration of treatment of lung. tuberculasis becomes also shorter. In countries where facilities are easily available, the duration of treaiment of lung tuberculowis is still 24 to 36 months. In countries with inadequate facilities and where the patient must be forced to get treatment, shortening in the sense of 6 to 12 months has been propased. Some workers clam that their shortening trials are successful by showing a low relapse rate.

Next to shortening of the period, an intermittent regimen (biweekly or 3 times a week) is proposed. No exact data of this latter regimen in Indonesia are available so far. It is hoped that a regimen of 6 to 12 months can be carried out with success in Indonesia rather than the intermittent schedule. 